\title{
0.7 Structure and Zero Bias Anomaly in Ballistic Hole Quantum Wires
}

\author{
R. Danneau, ${ }^{1,2, *}$ O. Klochan, ${ }^{1}$ W. R. Clarke, ${ }^{1}$ L. H. Ho, ${ }^{1}$ A. P. Micolich, ${ }^{1}$ M. Y. Simmons, ${ }^{1}$ \\ A. R. Hamilton, ${ }^{1}$ M. Pepper, ${ }^{3}$ and D. A. Ritchie ${ }^{3}$ \\ ${ }^{1}$ School of Physics, University of New South Wales, Sydney 2052, Australia \\ ${ }^{2}$ Low Temperature Laboratory, Helsinki University of Technology, Espoo, Finland \\ ${ }^{3}$ Cavendish Laboratory, J. J. Thomson Avenue, CB3 OHE Cambridge, United Kingdom
}

(Received 7 February 2007; published 10 January 2008)

\begin{abstract}
We study the anomalous conductance plateau around $G=0.7\left(2 e^{2} / h\right)$ and the zero bias anomaly in ballistic hole quantum wires with respect to in-plane magnetic fields applied parallel $B_{\|}$and perpendicular $B_{\perp}$ to the quantum wire. As seen in electron quantum wires, the magnetic fields shift the 0.7 structure down to $G=0.5\left(2 e^{2} / h\right)$ and simultaneously quench the zero bias anomaly. However, these effects are strongly dependent on the orientation of the magnetic field, owing to the highly anisotropic effective Landé $g$-factor $g^{*}$ in hole quantum wires. Our results highlight the fundamental role that spin plays in both the 0.7 structure and zero bias anomaly.
\end{abstract}

DOI: 10.1103/PhysRevLett.100.016403

One-dimensional (1D) systems have revealed some of the most intriguing effects in solid state physics [1]. For example, it is well-known that the Coulomb interactions in 1D electron systems lead to a correlated non-Fermi liquid state known as the Tomonaga-Luttinger liquid [1]. van Wees et al. [2] and Wharam et al. [3] demonstrated the underlying quantum nature of $1 \mathrm{D}$ noninteracting electronic systems, following the seminal work of Thornton et al. [4]. In these experiments it was shown that the conductance of a ballistic 1D system is quantized in units of $G_{0}=2 e^{2} / h$ corresponding to the discreteness of the number of propagating modes in the channel (see Ref. [5] for a review). This phenomenon has been studied extensively in 1D electron systems leading to the discovery of more exotic phenomena such as the unexpected feature around the unquantized value of $0.7 G_{0}$. This conductance anomaly was pointed out first in the early 1990s [6] and studied later in detail [7], the origin of which is still debated [8]. Because the noninteracting particle theory does not expect such a feature [5], this conductance anomaly, known as the 0.7 structure, is believed to be a manybody effect. In this work, we present a study of the 0.7 structure and its nonlinear counterpart, the zero bias anomaly (ZBA), in hole quantum wires, and demonstrate that both show an anisotropic response to an external magnetic field, which suggests that both are closely linked and related to spin.

The 0.7 structure is a resonance-like feature that sits below the first conductance plateau. It appears to be a zero-field remnant feature of the first spin-resolved plateau $e^{2} / h$ when the spin degeneracy is lifted by the Zeeman effect [7]. This picture is consistent with the fact that the 0.7 structure shifts to $e^{2} / h$ with the application of an inplane magnetic field. Temperature dependence studies showed that while the quantized plateaus are thermally smeared, the 0.7 structure becomes stronger [7,9]. This indicates that the 0.7 structure is not due to electron backscattering from a defect in the vicinity of the constriction
PACS numbers: 71.70.-d, 73.21.Hb, 73.23.Ad

[5], but is thermally activated and therefore not a ground state property [10]. In addition, the 0.7 structure coincides with an enhanced conductance at zero source-drain bias, which falls away rapidly as the bias between source and drain is increased. This conductance peak shares many of the features of the ZBA in quantum dots [11], including the splitting and ultimate destruction of the zero bias peak with the application of an in-plane magnetic field. This has led to a description of the 0.7 structure in terms of a Kondo-like correlated state within the constriction $[12,13]$.

While debate continues as to the exact origin of the 0.7 structure [7,12-17], the anomalous conductance plateau is commonly believed to be linked with spin. We therefore expect that the magnetic field dependence of both the conductance plateau and ZBA will strongly depend on the 1D effective Landé $g$-factor $g^{*}$, which determines how the spin couples with an external magnetic field. Here, we demonstrate this dependence explicitly by examining the magnetic field dependence of the 0.7 structure in hole quantum wires. Unlike electron 1D systems, which have an isotropic $g^{*}$, the $g$-factor in hole quantum wires is highly anisotropic [18]. We show that the conductance plateau and ZBA also share this anisotropy by examining their behavior with respect to in-plane magnetic fields applied parallel $B_{\|}$and perpendicular $B_{\perp}$ to the wire. In doing so we highlight the fundamental role that spin plays in these phenomena.

For these experiments, we used the 1D hole bilayer system grown on (311)A $n^{+}$-GaAs substrate described previously [19]. Electrical measurements were performed in the top wire of the bilayer hole system (the top layer has mobility $92 \mathrm{~m}^{2} \mathrm{~V}^{-1} \mathrm{~s}^{-1}$ and density $1.2 \times 10^{15} \mathrm{~m}^{-2}$ ) in a dilution refrigerator using standard low-frequency ac lockin techniques with an excitation voltage of $20 \mu \mathrm{V}$ at $17 \mathrm{~Hz}$. Side gates and a middle gate created by standard electron beam lithography define the quantum wires along the [233] direction. 


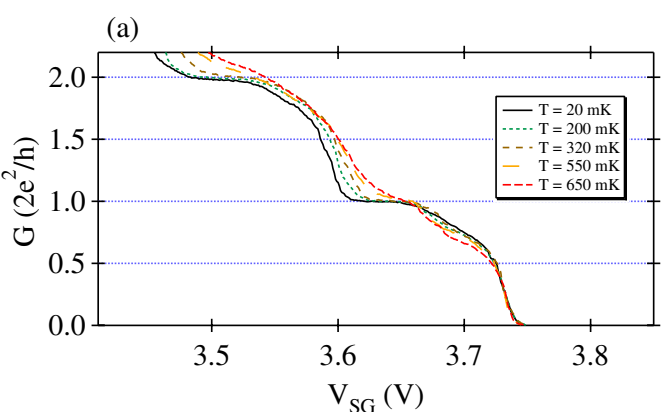

(b)

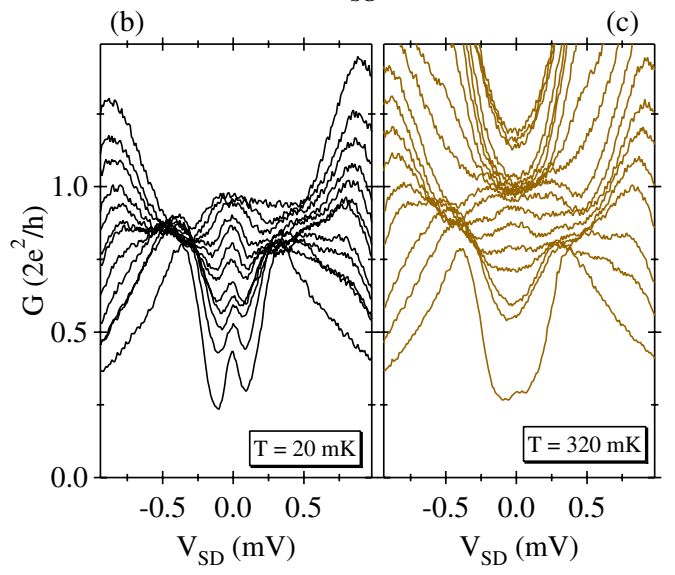

FIG. 1 (color online). (a) $G$ of the quantum wire versus sidegate voltage $V_{\mathrm{SG}}$, for $T=20,200,320,550$, and $650 \mathrm{mK}$; Each curve is shifted to sit on top of the $20 \mathrm{mK}$ curve; (b) $G$ versus source-drain bias $V_{\mathrm{SD}}$ for different $V_{\mathrm{SG}}$ at $T=20 \mathrm{mK}$; (c) $G$ versus $V_{\mathrm{SD}}$ for different $V_{\mathrm{SG}}$ at $T=320 \mathrm{mK}$; data are taken for back and middle gates fixed at 2.5 and $-0.225 \mathrm{~V}$ respectively. No magnetic field is applied to the system; (a), (b) and (c) are data taken from the same cool down.

In Fig. 1(a) we show the differential conductance $G=$ $d I / d V$ (corrected for a $2.5 \mathrm{k} \Omega$ series resistance) of the two first $1 \mathrm{D}$ subbands as a function of side-gate voltage $V_{\mathrm{SG}}$ for different temperatures. The anomalous plateau at $0.7 G_{0}$ becomes significantly stronger as the quantized plateaus are washed out with increasing temperature, as has been observed in induced hole quantum wires [20]. This indicates that the observed feature is not a conductance transmission resonance due to a defect in the vicinity of the constriction. The higher plateaus become unresolvable above $T \gtrsim 600 \mathrm{mK}$, i.e., at temperatures approximately an order of magnitude smaller than for 1D electron systems $[7,10]$. This is due to the greater effective mass of holes $m_{h}^{*} \approx 5 m_{e}^{*}$, which results in significantly smaller subband spacings in $p$-type 1D systems [19]. In Figs. 1(b) and 1(c) we also show the conductance as a function of source-drain bias (SDB) for several $V_{\mathrm{SG}}$. The data show a well-defined ZBA (the narrow peak in conductance around $V_{\mathrm{SD}}=0 \mathrm{~V}$ ) for conductances below $2 e^{2} / h$ [Fig. 1(b)]: For $T \approx$ $300 \mathrm{mK}$, the ZBA has vanished [Fig. 1(c)], a temperature that is approximately 2 times less than for electron systems $[12,21]$. These results are consistent with previous studies of spin-dependent focussing in 1D hole systems [22].
Once the 1D constriction is created, increasing $V_{\mathrm{SG}}$ moves the Fermi level through the 1D spin degenerate subbands [5]. An in-plane magnetic field lifts the spin degeneracy of the 1D subbands [3], with the nondegenerate subbands becoming quantized in units of $e^{2} / h$ above a critical field $B_{C}$. Figure 2(a) shows the effect of the inplane magnetic field parallel to the wire $B_{\|}$on the quantized steps and the 0.7 structure. We observe clearly the Zeeman splitting of the 1D subbands and the smooth evolution of the plateau at $0.7 G_{0}$ to $0.5 G_{0}=e^{2} / h$ at $B_{\|}=$ 3.6 T, i.e., to the complete spin-resolved conductance. Figure 2(b) shows a gray scale of the transconductance $d G / d V_{\mathrm{SG}}$ of the same data highlighting the shift of the 0.7 structure to $0.5 G_{0}$ around $B_{\|}=3.6 \mathrm{~T}$. After thermal cycling and reorientation of the sample, an in-plane magnetic field perpendicular to the constriction $B_{\perp}$ was applied. Figure 2(c), shows a gray scale of the transconductance at $T=20 \mathrm{mK}$ as a function of $G$ and in-plane magnetic
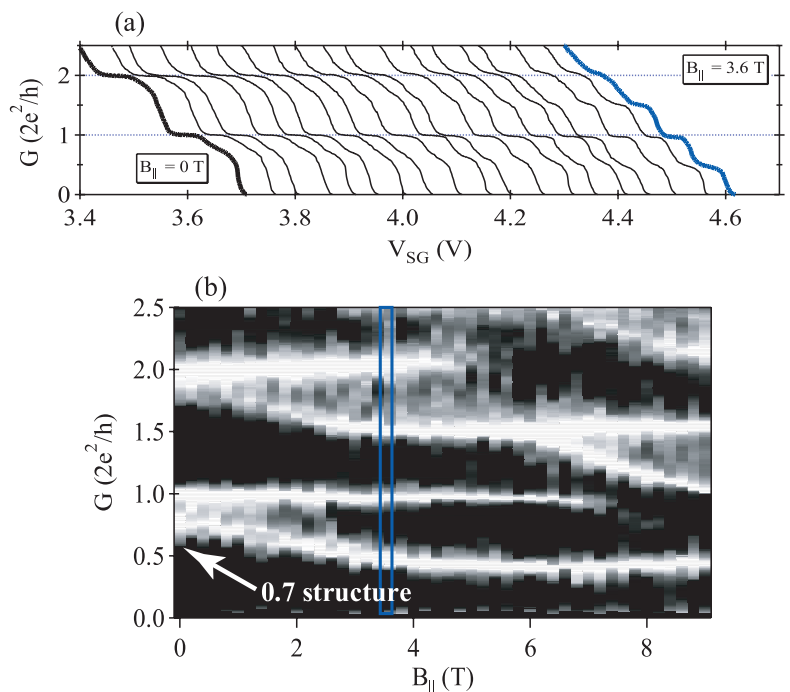

(c)

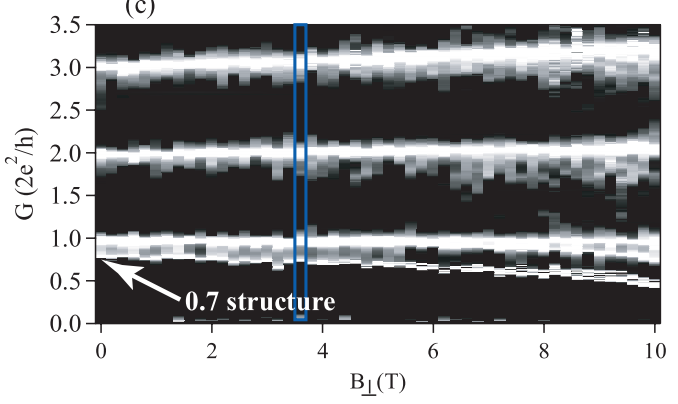

FIG. 2 (color online). (a) $G$ of the quantum wire versus sidegate voltage $V_{\mathrm{SG}}$ for different in-plane magnetic fields parallel to the wire $\left(B_{\|}\right)$from $0 \mathrm{~T}$ (i.e., when 1D subbands are degenerate), to $3.6 \mathrm{~T}$ (i.e., when $1 \mathrm{D}$ subbands are completely spin-resolved), in increments of $0.2 \mathrm{~T}$ (from left to right). $T=20 \mathrm{mK}$, back and middle gates are at 2.5 and $-0.225 \mathrm{~V}$, respectively. All curves are offset for clarity. (b) gray scale of the transconductance as a function of $B_{\|}$up to $8.8 \mathrm{~T}$ and $G$. White regions correspond to low transconductance (conductance plateaus). (c) gray scale of the transconductance as a function of versus $B_{\perp}$ and $G$, with similar experimental conditions. 
field $B_{\perp}$ up to 10 T. $B_{\perp}$ does not affect the quantized steps. This highlights the extreme anisotropy of the $g$-factor in 1D hole systems that has been observed previously [18]. This anisotropic behavior of the Zeeman splitting arises from the 1D confinement in this system with strong spinorbit (SO) coupling which forces the quantization axis of $\hat{J}$, originally perpendicular to the 2D plane [23], to align along the length of the wire [18].

Consistent with the behavior of the 0.7 structure, SDB measurements show that the ZBA exhibits the same remarkable anisotropy, demonstrating the peculiarities of holes compared to electrons. Figures 3(a) and 3(b) show the conductance as a function of SDB for $B_{\|}=0 \mathrm{~T}$ and $B_{\|}=3.6 \mathrm{~T}$ respectively. The clear ZBA measured at zero magnetic field is suppressed by $B_{\|}=3.6 \mathrm{~T}$, coinciding with the conductance plateau reaching $G=0.5 G_{0}$ and fully spin-resolved of higher subbands. The magnetic field dependence is significantly different for the $B_{\perp}$ case. Figures 3(c)-3(e) present SDB data from for $B_{\perp}=0$, 3.6, and $10 \mathrm{~T}$, respectively, taken during the same cool down as in Fig. 2(c). The clear and reproducible ZBA remains intact at $B_{\perp}=3.6 \mathrm{~T}$ and does not seem to be affected by the magnetic field. At $B_{\perp}=10 \mathrm{~T}$, the ZBA is finally destroyed.

Confinement and SO coupling alter drastically the hole properties in zinc-blende compounds. The $2 \mathrm{D}$ confinement lifts the heavy hole $(\mathrm{HH})$-light hole $(\mathrm{LH})$ degeneracy with HH's occupying the lowest energy state. This means that the carrier transport is predominantly via the HH subband [23]. It is therefore surprising that the 0.7 structure evolves smoothly to $0.5 G_{0}$ with increasing $B_{\perp}$ despite no evidence for Zeeman splitting in the higher subbands. Nonetheless, its evolution is still highly anisotropic. For the $B_{\|}$case, the 0.7 structure falls rapidly, reaching $0.5 G_{0}$ at $B_{\|}=3.6 \mathrm{~T}$. On the contrary, the 0.7 structure is barely affected at $B_{\perp}=$ $3.6 \mathrm{~T}$ and reaches $0.5 G_{0}$ only at much larger magnetic fields $B_{\perp}=10 \mathrm{~T}$. We notice that the evolution of the 0.7 structure is complicated at very high fields by a weak additional feature in the conductance that appears to split off from the 1D subband [24]. However, SDB measurements at $B_{\perp}=10 \mathrm{~T}$ reveal that the ZBA is absent at this field, suggesting that 0.7 structure has indeed reached its spin-resolved state (i.e., $0.5 G_{0}$ ). This highly anisotropic behavior with respect to the in-plane magnetic field reinforces strongly the hypothesis that the 0.7 structure and the ZBA are linked and spin-related phenomena. However, the question still remains as to why we observe any effect of $B_{\perp}$ on the 0.7 structure and ZBA at all up to $3.6 \mathrm{~T}$, a magnetic field sufficiently large that it leads to a fully spin-resolved state in the parallel orientation?

The higher subbands show little evidence of spin splitting up to $B_{\perp}=10 \mathrm{~T}$, indicating that the $g_{\perp}^{*}$ for the first subband is at least 4.5 times less than $g_{\|}^{*}[18]$; i.e., $g_{\|}^{*} / g_{\perp}^{*}>$ 4.5. On the other hand, the shift in the 0.7 structure and the destruction of the ZBA at $B_{\perp}=10 \mathrm{~T}$ suggest that these features are more sensitive to an external magnetic field. In addition, if we compare the rate at which the 0.7 structure evolves toward $0.5 G_{0}$ as a function of $B_{\|}$and $B_{\perp}$ we find that the anisotropy can be no greater than $g_{\|}^{*} / g_{\perp}^{*} \leq 4$. This reduction in the anisotropy is also consistent with calculations based on our device structure (i.e., quantum well width $W_{x}$ and 2D hole density) and crystallographic orientation [25]. The calculations predict that 1D confinement induced HH-LH band mixing results in a significant enhancement of both $g_{\|}^{*}$ and $g_{\perp}^{*}$. However, $g_{\perp}^{*}$ is predicted to increase more rapidly with confinement, resulting in a reduction in the $g$-factor anisotropy: our measurements are consistent with this. With increasing confinement, the $g$-factor anisotropy is predicted to become zero and then ultimately reverse in the extreme 1D limit where the width of the wire $W_{y}$ becomes comparable to the width of the quantum well $W_{x}$, i.e., $g_{\perp}^{*}>g_{\|}^{*}$ for $W_{x} / W_{y} \gtrsim 0.42$. The continuous transition in $g$-factor anisotropy corresponds to a monotonic change in the carriers from $\mathrm{HH}$ character in

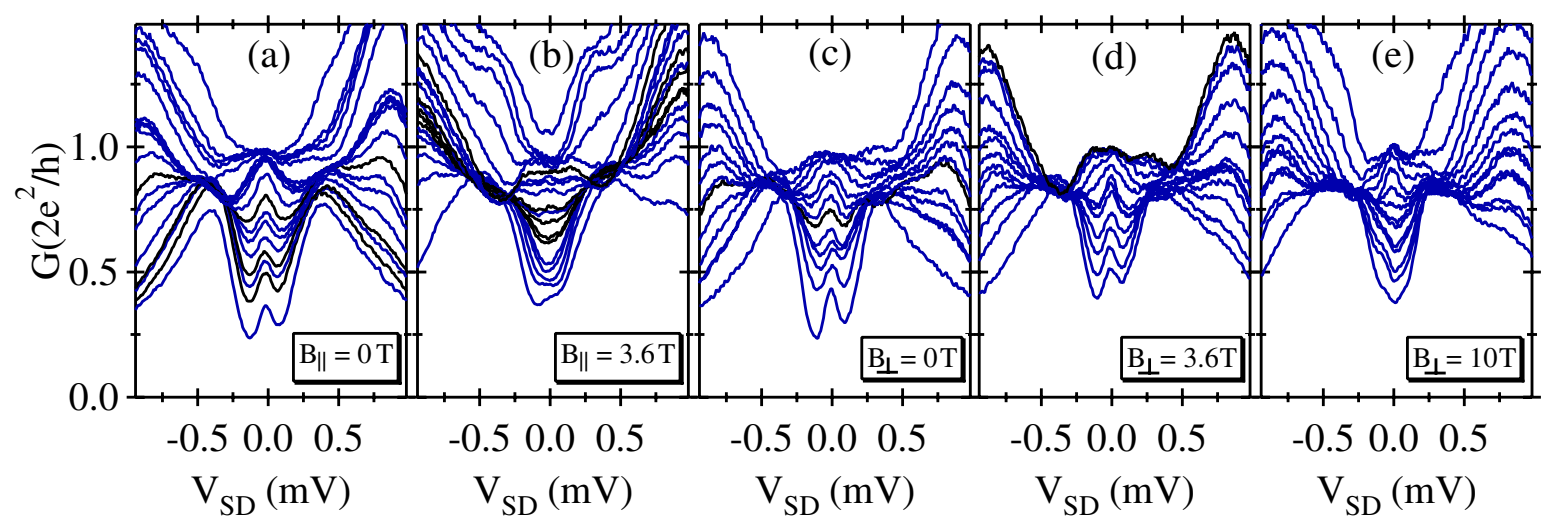

FIG. 3 (color online). (a) and (b): $G$ versus $V_{\mathrm{SD}}$ for different $V_{\mathrm{SG}}$ of the same quantum wire at (a) $B_{\|}=0 \mathrm{~T}$ and (b) $B_{\|}=3.6 \mathrm{~T}$, $T=20 \mathrm{mK}$, back and middle gates are at 2.5 and $-0.225 \mathrm{~V}$, respectively, [(a) and (b) are taken at the same cool down]. (c), (d), and (e): $G$ versus $V_{\mathrm{SD}}$, for different $V_{\mathrm{SG}}$, for $B_{\perp}=0,3.6$ and $10 \mathrm{~T}$, in similar experimental conditions [(c), (d) and (e) are taken at the same cool down; (c) and Fig. 1(b) are the same data]: the ZBA is clearly observed at $B_{\perp}=0$ and $3.6 \mathrm{~T}$. At $B_{\perp}=10 \mathrm{~T}$, the ZBA vanishes at low conductance. 
the 2D limit to $\mathrm{LH}$ character in the symmetric wire limit $W_{y}=W_{x}$, which results from confinement induced $\mathrm{HH}-$ LH band mixing.

The extreme 1D limit is inaccessible in our samples due to the long Fermi wavelength $\lambda_{F}$. At the first plateau $W_{y}=$ $\lambda_{F}^{1 \mathrm{D}} / 2$ and we expect $\lambda_{F}^{1 \mathrm{D}}$ to be approximately 4 times greater than the $2 \mathrm{D}$ Fermi wavelength [26], i.e., $\lambda_{F}^{1 \mathrm{D}} / 2 \approx$ $140 \mathrm{~nm}$ giving $W_{x} / W_{y} \approx 0.15$ [27]. However, even at this level of confinement we can expect to see the effects of band mixing below the first subband [25] and hence a reduction in $g$-factor anisotropy for the 0.7 structure [28] - as observed in our experiments. We note that since the magnetic length $l_{B}=\sqrt{\hbar / e} B$ can be comparable to the width of the wire even at moderate fields, magnetic confinement may contribute to the $g$-factor anisotropy at large $B$ (in addition to LH-HH mixing). However, the anisotropic behavior of the 0.7 structure at low fields is unlikely due to magnetic confinement. Furthermore, since we compare the measured anisotropy of the ZBA and the measured behavior of the 0.7 structure, the conclusion that both conductance anomalies are spin-related is still valid, even with strong SO coupling.

There is not yet a consensus as to the origin of the 0.7 structure. Some of the theories include spontaneous spin polarization [14], Kondo effect [13], electron-phonon scattering [15], charge density waves [16], or Wigner crystals [17]. While a detailed comparison of our data with these various models is beyond the scope of this work, our data provide valuable new constraints on theory: while interactions are strongly enhanced due to the large effective mass, holes have an effective spin $J=3 / 2$, so the spin-physics could be different.

To summarize, we have studied the 0.7 structure and the ZBA in 1D hole systems. We have shown that both features react to an in-plane magnetic field depending on its orientation with respect to the quantum wire direction. These findings strongly support, first that the 0.7 structure and ZBA are directly linked, and second that they are both related to spin. Finally, we have shown that the 0.7 structure evolves slowly to $0.5 G_{0}$ despite the suppression of the Zeeman splitting for $\mathrm{HH}$ when an in-plane magnetic field is applied perpendicular to the wire; this may be the result of $\mathrm{HH}-\mathrm{LH}$ mixing induced by the 1D confinement.

We wish to acknowledge U. Zülicke for enlightening discussions and J. W. Cochrane for technical support. R. D. and A. P. M. acknowledge additional support of ARC postdoctoral grants. M. Y.S. and A.R.H. acknowledge additional support of an ARC Federation grant and an ARC Professorial grant, respectively. This work was funded by the ARC and the EPSRC.

*Corresponding author. r.danneau@boojum.hut.fi
[1] T. Giamarchi, Quantum Physics in One Dimension (Oxford University Press, New York, 2003).

[2] B. J. van Wees et al., Phys. Rev. Lett. 60, 848 (1988).

[3] D. A. Wharam et al., J. Phys. C 21, L209 (1988).

[4] T. J. Thornton et al., Phys. Rev. Lett. 56, 1198 (1986).

[5] C. W. J. Beenakker and H. van Houten, Solid State Phys. 44, 1 (1991).

[6] N. K. Patel et al., Phys. Rev. B 44, 13549 (1991).

[7] K. J. Thomas et al., Phys. Rev. Lett. 77, 135 (1996).

[8] G. Fitzgerald, Phys. Today 55, No. 5, 21 (2002).

[9] B. J. van Wees et al., Phys. Rev. B 43, 12431 (1991).

[10] A. Kristensen et al., Phys. Rev. B 62, 10950 (2000).

[11] D. Goldhaber-Gordon et al., Nature (London) 391, 156 (1998); S. M. Cronenwett, T. H. Oosterkamp, and L.P. Kouwenhoven, Science 281, 540 (1998); J. Nygård, D. H. Cobden, and P.E. Lindelof, Nature (London) 408, 342 (2000).

[12] S. M. Cronenwett et al., Phys. Rev. Lett. 88, 226805 (2002).

[13] P. E. Lindelof, SPIE Int. Soc. Opt. Eng. 4415, 77 (2001); Y. Meir, K. Hirose, and N. S. Wingreen, Phys. Rev. Lett. 89, 196802 (2002); K. Hirose, Y. Meir, and N.S. Wingreen, Phys. Rev. Lett. 90, 026804 (2003); P. S. Cornaglia and C. A. Balseiro, Europhys. Lett. 67, 634 (2004); T. Rejec and Y. Meir, Nature (London) 442, 900 (2006); A. Golub, T. Aono, and Y. Meir, Phys. Rev. Lett. 97, 186801 (2006).

[14] C.-K. Wang and K.-F. Berggren, Phys. Rev. B 54, R14257 (1996); L. Calmels and A. Gold, Europhys. Lett. 39, 539 (1997); D. J. Reilly et al., Phys. Rev. Lett. 89, 246801 (2002).

[15] G. Seelig and K. A. Matveev, Phys. Rev. Lett. 90, 176804 (2003).

[16] O. P. Sushkov, Phys. Rev. B 64, 155319 (2001).

[17] B. Spivak and F. Zhou, Phys. Rev. B 61, 16730 (2000); K. A. Matveev, Phys. Rev. Lett. 92, 106801 (2004); Phys. Rev. B 70, 245319 (2004); A. D. Klironomos et al., Phys. Rev. B 76, 075302 (2007).

[18] R. Danneau et al., Phys. Rev. Lett. 97, 026403 (2006).

[19] R. Danneau et al., Appl. Phys. Lett. 88, 012107 (2006).

[20] O. Klochan et al., Appl. Phys. Lett. 89, 092105 (2006).

[21] The width of the ZBA at $G_{0} / 2$ is about $100 \mu \mathrm{V}$, significantly less than in [12].

[22] L. P. Rokhinson, L. N. Pfeiffer, and K. W. West, Phys. Rev. Lett. 96, 156602 (2006).

[23] R. Winkler, Spin-Orbit Coupling Effects in TwoDimensional Electron and Hole Systems (Springer, Berlin, 2003).

[24] We notice that similar weak features appear at high magnetic field in Ref. [22].

[25] U. Zülicke, Phys. Status Solidi (c) 3, 4354 (2006).

[26] J. G. Williamson et al., Phys. Rev. B 42, 7675 (1990); R. Crook et al., J. Phys. Condens. Matter 12, L735 (2000).

[27] HH-LH band mixing appears around the first plateau, i.e., for $W_{x} / W_{y} \approx 0.15$, ultimately causing the LH to become the ground state of the system.

[28] Note that the reduction in the $g$-factor anisotropy for the 0.7 structure does not mean that the behavior of either $g_{\|}^{*}$ or $g_{\perp}^{*}$ in nonmonotonic, but that $g_{\|}^{*}$ and $g_{\perp}^{*}$ may be changing at different rates as the wire is made narrower. 